ARTICLE

\title{
Monte Carlo Simulation of Prompt Neutron Evaporation by Fragments in Low Energy Nuclear Fission
}

\author{
Djelloul BENZAID ${ }^{1, *}$, Mohamed DJEBARA ${ }^{2}$ and Abdeslam SEGHOUR ${ }^{3}$, \\ ${ }^{1}$ Centre Universitaire de Khemis Miliana, Route de Theniat El Had BP 44225, Ain Defla, Algeria \\ ${ }^{2}$ Université des Sciences et de la Technologie Houari Boumediene, Alger, Algeria \\ ${ }^{3}$ Centre de Recherche Nucléaire d'Alger, Algeria
}

\begin{abstract}
In this work, we performed a Monte Carlo simulation of the evaporation of prompt neutron in the fission at low energy. We are interested in the reaction ${ }^{249} C f\left(n_{t h}, f\right)$ we have a detailed experimental study of isotonic distributions at different kinetic energies. The statistical Fong model is used to calculate the intrinsic excitation energy shared between the two fragments of fission. The simulation reproduces correctly mass and isotonic distributions. However, only the peak located at $\mathrm{N}=59$ of the local even-odd effect has been found.
\end{abstract}

KEYWORDS: Monte-Carlo, Neutron, even-odd effect, nuclear fission

\section{Introduction}

In this work we have simulated the process of evaporation of prompt neutrons using the Monte-Carlo method, in order to provide possible explanations to some experimental facts by simulating the phase between the excited state of the compound nucleus and the end of the evaporation of neutrons by the fragments, a phase that is practically impossible to detect experimentally. We go back to the primary mass distributions, i.e., before evaporation of neutrons, from the measured charge distributions of the reaction, representing the fragments at the scission phase. ${ }^{1)}$

The potential energy of deformation $P$ is calculated in the framework of the liquid drop model, ${ }^{2)}$ and the sharing of intrinsic excitation energy is done using the Fong mode, ${ }^{3)}$ assuming that the two fragments are at the same temperature, while minimizing $P$ with respect to deformation parameters $\alpha_{31}$ and $\alpha_{32}$. In our simulation the evaporation of neutrons is supposed certain once the total excitation energy of fragment $E_{e x}$ is greater than the sum of the evaporated neutron separation energy $B_{n}$ and its kinetic energy $\varepsilon_{n}$ which is assumed to follow a maxwellian distribution.

In this work we discuss the effect of evaporation of neutrons on different observables, in particular isotonic initial distribution. We also try to see to what extent the observed structures in the local even-odd effect can be or not related to the process of evaporation.

\section{Even-Odd Effect}

The charge distribution represents the charge measured at the scission point since there is no proton evaporation. The measurement of this observable is an effective means of in-

*Corresponding author, E-mail:benzaidhd@yahoo.com

(a) AdomicaEmerigyeserigtssofiłaparf Japan, All Rights Reserved. vestigating the scission point

The charge even-odd effect is defined as:

$$
\delta_{p}=\frac{Y_{e}-Y_{o}}{Y_{e}+Y_{o}} \times 100(\%)
$$

where: $Y_{o}$ and $Y_{e}$ represents respectively, the yields of all charges in the odd and even charge distribution of fragments.

We also define similarly the neutron pairing effect $\delta_{n}$.

Unlike the charge distributions, the pairing effect of neutrons represents the final isotonic distribution since it is measured after evaporation of neutrons.

\section{Simulation of Neutron Evaporation Process Using Monte-Carlo Method}

The process of evaporation of prompt neutrons in fission at low energy of ${ }^{249} \mathrm{Cf}\left(n_{t h}, f\right)$ is simulated by the Monte-Carlo method. This method is essentially based on the random drawing of quantities, such as the number of initial mass, evaporated neutron energy and the excitation energy of fission fragments, following distributions pre-selected on the basis of experimental results and if necessary assumptions. Our simulated results are compared with those obtained by Djebara et al. ${ }^{11}$

The path of fission starts from by absorption of the target nucleus of a thermal neutron and ends with the fragmentation of the formed compound nucleus into two lighter fragments in excited states.

Our simulation program is based on the following key elements:

1. Determination of yields of primary masses from experimental charge yields by drawing random mass of the fragment following a gaussian distribution.

2. Potential energy is calculated from the liquid drop 


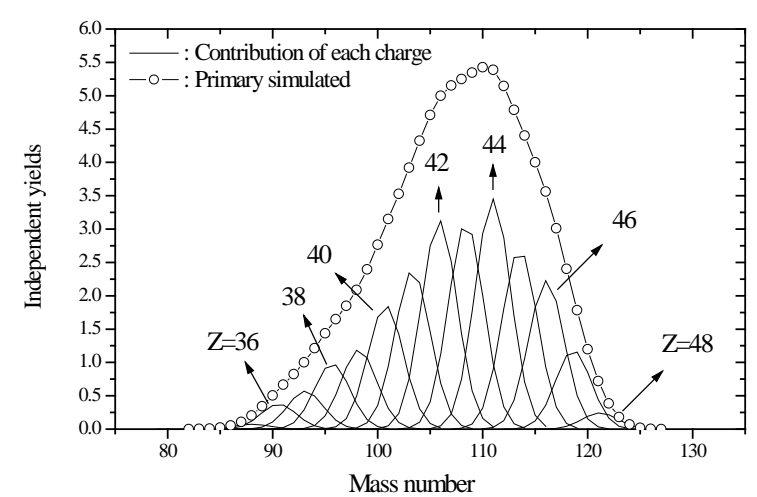

Fig. 1 Experimental isotopic distribution

model minimizing it with respect to deformation parameters $\alpha_{31}$ and $\alpha_{32}$.

3. Total excitation energy is shared between the two fragments using the model of Fong. The energy released $Q$ is calculated from the mass table of Audi and Wapstra. ${ }^{4)}$

4. Simulation of evaporation according the model of Fong. ${ }^{3)}$

\section{Definition of Different Important Quantities}

\section{Primary Mass Yields}

The primary mass distributions are constructed from the measured charge distributions (Fig. 1) by drawing random mass of light fragment following a gaussian distribution :

$$
Y\left(A^{\prime} \mid Z\right)=\frac{Y(Z)}{\sigma_{A} \sqrt{2 \pi}} \exp \left[-\frac{\left(A^{\prime}-\bar{A}\right)^{2}}{2 \sigma_{A}^{2}(Z)}\right]
$$

where: $Y(Z)$ and $\sigma_{A}$ represent, respectively, experimental charge distribution and the standard deviation of the distribution and is given by:

$$
\sigma_{A}(Z)=0.04 Z+0.20
$$

$\bar{A}$ is the mean of mass number before evaporation of neutrons. It is given by UCD ${ }^{\mathrm{a}}$ hypothesis as:

$$
\bar{A}=\frac{A_{F}}{Z_{F}}(Z-0.5)
$$

$A_{F}$ and $Z_{F}$ are, respectively, the mass and charge of the nucleus undergoing fission.

The primary yields are obtained simply by adding all isotopic contributions:

$$
Y\left(A^{\prime}\right)=\sum_{i} Y\left(A^{\prime} \mid Z_{i}\right)=\sum_{i} \frac{Y(Z)}{\sigma_{A} \sqrt{2 \pi}} \exp \left[-\frac{\left(A^{\prime}-\bar{A}\right)^{2}}{2 \sigma_{A}^{2}(Z)}\right]
$$

Figure 2 represents simulated total and independent mass yields.

\footnotetext{
${ }^{a}$ UCD hypothesis (Unchanged Charge Density) supposes that the ratio $(Z / A)$ remains unchanged for both of nuclei undergoing fission and the two fragments of fission before evaporation.
}

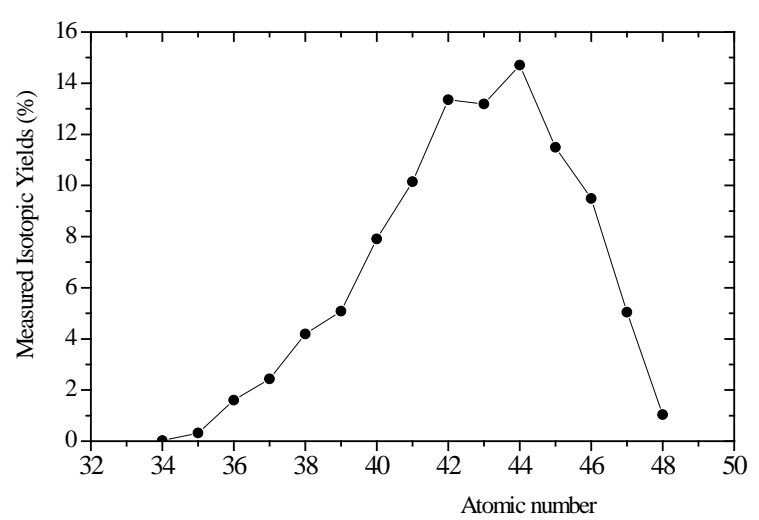

Fig. 2 Independent and total simulated mass distributions

\section{Potential Energy}

The motion of a point on the nuclear surface can be described by a series expansion of legendary polynomials:

$$
R(\theta)=R_{0}\left[1+\alpha_{2} P_{2}(\cos \theta)+\alpha_{3} P_{3}(\cos \theta)+\cdots\right]
$$

Assuming that the deformation is most likely octupole deformation, we can content ourselves with the term $P_{3}$ in the previous development. In this case, the mutual Coulomb energy of two fragments is given as a function of the parameters of deformation, by the expression:

$$
C\left(\alpha_{31}, \alpha_{32}\right)=\frac{Z_{L} Z_{H} e^{2}}{R_{0 L}\left(1+0.9314 \alpha_{3 L}\right)+R_{0 H}\left(1+0.9314 \alpha_{3 H}\right)}
$$

where $R_{0 L}$ and $R_{0 H}$ represent the radius of the light and heavy fragments respectively. These are given by:

$$
R_{0}=r_{0} A^{1 / 3}
$$

with $r_{0}=1.5$ Fermi

As for the deformation energies, if we limit ourselves to terms $P_{3}$, they can be calculated from the expression:

$$
D_{i}\left(\alpha_{3 i}\right)=0.7143 \alpha_{3 i}^{2} E_{S i}(0)-0.2041 \alpha_{3 i}^{2} E_{C i}(0)
$$

with $i \equiv L, H$, and where $E_{S i}(0)$ and $E_{C i}(0)$ represent, respectively, the surface energy and Coulomb energy of the fragment and are given according to the model of the liquid drop model by:

$$
\begin{aligned}
& E_{S i}(0)=0.014 A^{2 / 3} a m u \\
& E_{C i}(0)=0.000627 \frac{Z_{i}^{2}}{A_{i}^{1 / 2}} a m u
\end{aligned}
$$

The potential energy is, then, given by:

$$
P\left(\alpha_{31}, \alpha_{32}\right)=C\left(\alpha_{3 L}, \alpha_{3 H}\right)+D\left(\alpha_{3 L}\right)+D\left(\alpha_{3 H}\right)
$$

\section{Sharing of Excitation Energy between the Heavy and Light Fragments}

Let $G$ be the total intrinsic excitation energy of the compound nucleus formed by the thermal incident neutron and the fissile nucleus, it is defined as the difference between the energy release $Q$ and the potential energy of the compound 


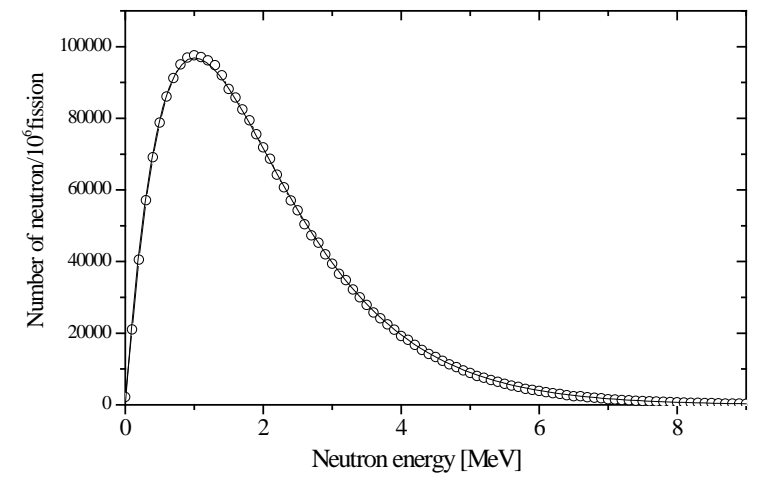

Fig. 3 evaporated neutron energy distribution

nucleus:

$$
G=Q-P
$$

The internal excitation energy corresponds most likely to the minimum of potential energy. The problem is therefore to minimize $P\left(\alpha_{31}, \alpha_{32}\right)$ with respect to the parameters of deformation $\alpha_{31}$ and $\alpha_{32}$.

Substituting Eqs. (6) and (8) by Eq. (11) one can obtain the potential energy expressed as a function of the parameters $\alpha_{31}$ and $\alpha_{32}$. On the other hand, it is assumed that the configuration at the scission point is that which corresponds to the minimum potential energy and thus to the maximum of the excitation energy, $E_{e x L}$ :

$$
\frac{\partial P\left(\alpha_{31}, \alpha_{32}\right)}{\partial \alpha_{31}}=0 \text { and } \frac{\partial P\left(\alpha_{31}, \alpha_{32}\right)}{\partial \alpha_{32}}=0
$$

Energy $G$ is the sum of the energies of the two fragments:

$$
G=G_{L}+G_{H}
$$

such that $G_{L}$ and $G_{H}$ are the intrinsic excitation energy of light and heavy fragments, respectively.

The two fragments are formed in contact; their temperatures should be the same, $T \sim 1 \mathrm{MeV}$. According to the statistical model of the nucleus, ${ }^{5)}$ we have:

$$
\frac{G_{L}}{G_{H}}=\frac{A_{L}}{A_{H}}
$$

We finally obtain the total excitation energy $E_{e x L}$ of the light fragment by adding the intrinsic excitation energy $G_{L}$ and the deformation energy $D_{L}$ and finally:

$$
E_{e x L}=G_{L}+D_{L}
$$

This energy will allow to simulate the evaporation of neutrons from light fragments, and thus determines the mass distribution and the final isotonic distributions and other important quantities.

\section{Neutron Emission}

The distribution of kinetic energies of evaporated neutrons is assumed to follow a maxwelienne distribution with nuclear temperature $T$ of around $1 \mathrm{MeV}$. The kinetic energy of a neutron evaporated is drawn, then, randomly following

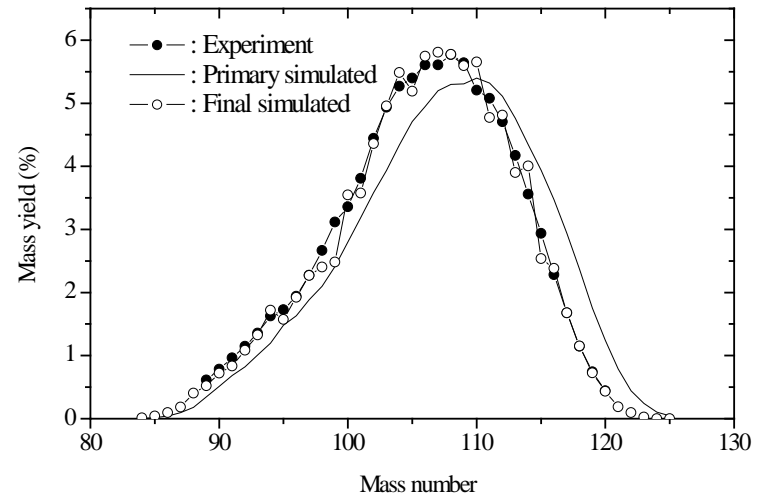

Fig. 4 Mass yield

a maxwelienne. The neutrons can be evaporated if the following condition is satisfied:

$$
E_{e x L}>B_{n}+\varepsilon_{n}
$$

where $\varepsilon_{n}$ and $B_{n}$ represent, respectively, the kinetic energy of evaporated neutron and the separation energy.

The distribution of neutron energies obtained by our simulation (Fig. 3) can be represented by the following maxwelienne formula:

$$
N\left(\varepsilon_{n}\right)=2.63 \times 10^{5} \varepsilon_{n} \exp \left(-\frac{\varepsilon_{n}}{T}\right)
$$

with $T \approx 1 \mathrm{MeV}$.

Note that the most probable value of energy of neutrons is around $1 \mathrm{MeV}$ and is identical to the experimental value showing a satisfactory drawing of these quantities.

\section{Results and Discussions}

\section{Final Mass Distribution}

The final mass distribution integrated over the kinetic energy is obtained after evaporation of neutrons. Figure 4 represents the result of our simulation. Yields are normalized to $100 \%$.

We have introduced the primary mass distribution for comparison. Note that the distribution after evaporation of neutrons is shifted to lighter masses. This shift is more important for the portion of the high masses than low ones. A fact which is due to the increased number of neutrons evaporated with the mass number $A$. This implies both that the width of the final distribution is smaller than the initial distribution, and secondly the maximum final distribution increases slightly from the initial distribution. The surface distribution is always maintained.

\section{Isotonic Distribution}

Isotonic distributions integrated over kinetic energy is obtained after adding all independent yields of fragments having the same number of neutrons $N$ :

$$
Y(N)=\sum_{A} Y(A, N)
$$

We have shown in Fig. 5 simulated and measured neutron distributions, we have also introduced, for comparison, the primary distribution. Note that the experimental distribution 


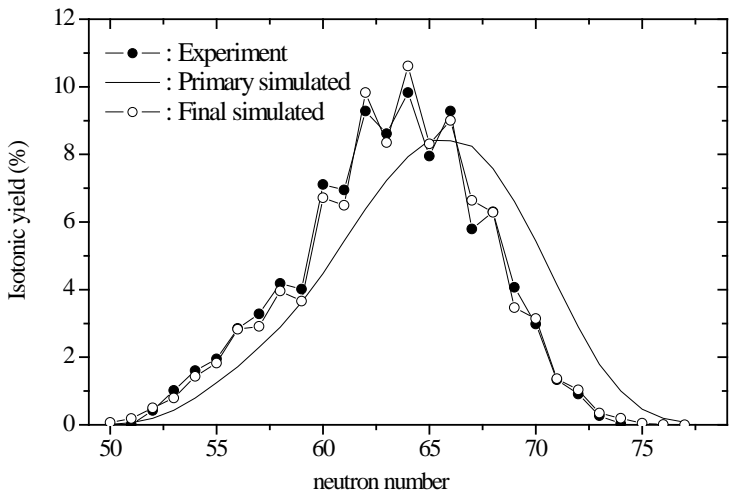

Fig. 5 Isotonic yield

is well reproduced by our simulation.

We also note the great similarity between the experimental curve and simulated one that are very structured, fragments with an even number $(N)$ of neutrons are more advantaged than those with neighbors odd number of neutrons $(N \pm 1)$.

The favored production of even isotones compared to odd ones is taken into account by the average even-odd effect given by:

$$
\delta_{n}=\frac{Y_{e}-Y_{o}}{Y_{e}+Y_{o}} \times 100(\%)
$$

where $Y_{e}$ and $Y_{o}$ represent the sum of all even and odd isotones yield in the isotonic distribution of fragments.

The neutron even-odd, calculated according to Eq. (20) is equal to $11 \%$, a value to be compared to the experimental value $(9.5 \pm 0.7) \%$.

\section{Average Number of Evaporated Neutrons}

Figure 6 represents a comparison between the evolution of the number of neutrons evaporated by fission fragments to that obtained by our simulation. The agreement is satisfactory; the general shape of the experimental spectrum is reproduced except that the simulated values are slightly lower than the experimental values. The disagreement is more important around the mass $A=120$.

\section{Local Even-Odd Effect}

For more detailed information, we can study the so called local even-odd effect using Tracy method. ${ }^{6)}$ This method consist of estimating the local even-odd effect by the following formula:

$$
d_{3}\left(N+\frac{3}{2}\right)=\exp \left\{(-1)^{N+1}\left[\left(L_{3}-L_{0}\right)-3\left(L_{2}-L_{1}\right)\right]\right\}
$$

$L_{0}, L_{1}, L_{2}$ and $L_{3}$ are the natural logarithms of isotonic yields $N, N+1, N+2, N+3$. On this interval, the even-odd effect can be estimated by the previous formula.

We represent in Fig. 7 the simulated and the measured local even-odd effect. The experimental peak located at $N \sim$ 60 is reproduced in an acceptable manner. A fact which suggests that the peak may be caused to evaporation of neutrons.

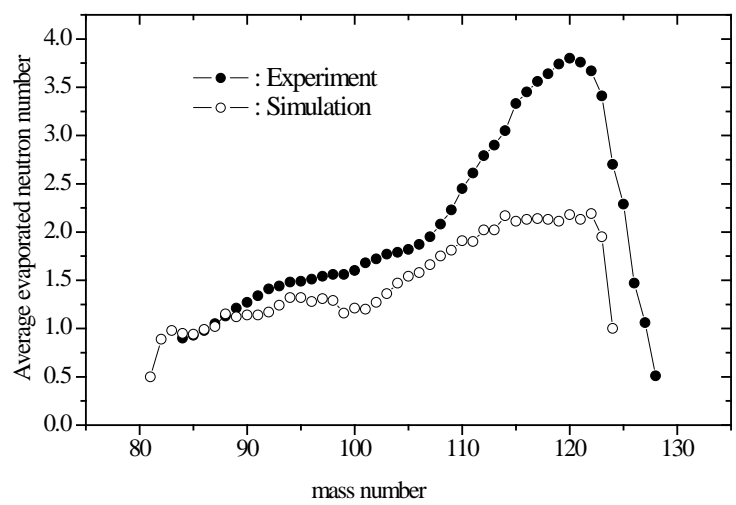

Fig. 6 Average evaporated neutron number

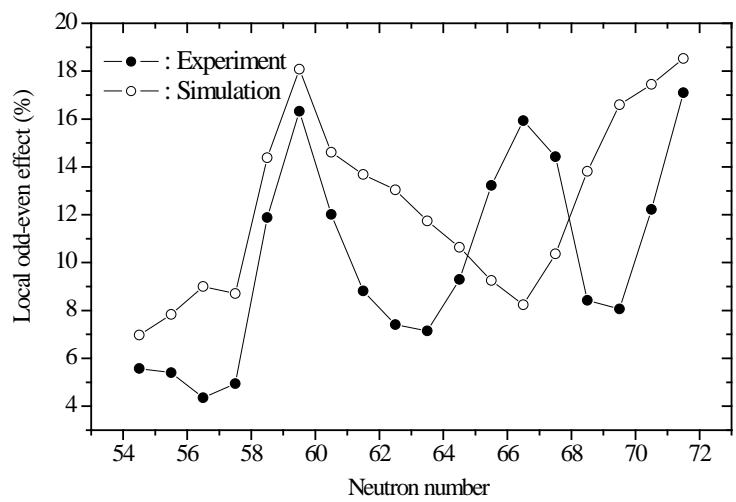

Fig. 7 Average evaporated neutron number

\section{Conclusion}

In our work we have simulated the process of evaporation of neutrons by light fragments of fission. We used the model of Fong to calculate the excitation energy and its partition between the two fragments. The yields of primary masses are constructed assuming that the yield of for each partial charge Z follow a Gaussian distribution. Mass and neutron yields are reproduced in a very satisfactory way. Neutron even-odd simulated is about $11 \%$. The peak located at $\mathrm{N}$ 60 in the local even-odd effect is reproduced. Thus, we conclude that it is due to evaporation of neutrons. The second peak is not reproduced. It can not be combined with a simple neutron evaporation.

\section{References}

1) M. Djebara, Mass, nuclear charge and kinetic energy distributions of fragments of fission of ${ }_{98}^{249} C f\left(n_{\text {th }}, f\right)$ and ${ }_{98}^{229} T h\left(n_{t h}, f\right)$, Doctorate dissertation, USTHB (1994), [In French].

2) N. Bohr, J. A. Wheeler, Phys. Rev., 56, 426-450 (1939).

3) P. Fong, Phys. Rev., 102, 434-448 (1956).

4) G. Audi ,A. H. Wapstra, Nucl. Phys., A595, 409-480 (1995).

5) R. R. Roy, B. P. Nigam, Nuclear Physics, theory and experiment, John Wiley and sons (1993).

6) B. L. Tracy, J. Chaumont, R. Klapisch, J. M. Nitschke, A. M. Poskanzer, E. Roeckl, C. Thibault, Phys. Rev., C5, 222-234 (1972). 